\title{
O EFEITO PAÍS SOBRE O DESEMPENHO DA FIRMA: UMA ABORDAGEM MULTINIVEL
}

\section{RESUMO}

Este trabal ho analisou a influência do país sobre o desempenho das firmas. A análise empírica tomou por base uma amostra de 83.641 observações de 10.927 firmas em 37 países e 224 indústrias, em um período de 10 anos. Um modelo linear hierárquico de três níveis com classificação cruzada permitiu identificar que a importância relativa dos efeitos país, indústria e interação indústria-país é semelhante e, em conjunto, aproximadamente da mesma magnitude do efeito firma. Além de estimar a importância do efeito país, o trabalho analisou a influência individual de cada país sobre o desempenho das firmas, o que permitiu a elaboração de um ranking de países com base na lucratividade das firmas.

\section{Rafael G. Burstein Goldszmidt}

FGV-EAESP

\section{Luiz Artur Ledur Brito}

FGV-EAESP

\section{Flavio Carvalho de Vasconcelos}

FGV-EAESP

ABSTRACT This paper analyses country effects on firm performance. Empirical analysis was based on a sample of 83,641 observations and 10,927 firms in 37 countries and 224 industries, in a 10 year time span. A cross-classified 3-level hierarchical linear model allowed the estimation of country, industry and country-industry inter action effects, which had approximately the same relative importance. Altogether, these effects were nearly as important as firm effect. The influence of each specific country on the performance of its firms was also studied and a ranking of countries based on firm profitability was developed.

PALAVRAS-CHAVE Estratégia, desempenho, vantagem competitiva, efeito país, modelos multinível.

KEMORDSStrategy, performance, competitive advantage, country effect, multilevel models. 


\section{INTRODUÇÃO}

A busca das fontes do desempenho superior de algumas firmas é um tema central na pesquisa em estratégia. Uma linha de pesquisa sobre os componentes de variância do desempenho tem oferecido importante apoio empírico nessa busca.

Embora a origem dessa linha remonte ao trabal ho de Schmalensee (1985), ela foi consolidada pelo trabalho seminal de Rumelt (1991), que ocupa uma posição central na literatura do campo da gestão estratégica (RamosRodríguez e Ruiz-N avarro, 2004). Ao trabal ho de Rumelt (1991) seguiu-se uma série de outros, utilizando amostras mais abrangentes e aprimoramentos metodológicos, que analisaram os efeitos firma, corporação, indústria e ano (Roquebert, Phillips e Westfall, 1996; McGahan e Porter, 1997; Brush e Bromiley, 1997; Brush, Bromiley e Hendrickx, 1999; Bowman e Helfat, 2001).

0 efeito firma foi a mais relevante fonte de variação no desempenho, seguido pelos efeitos indústria e corporação, com uma fração da variância muito inferior associada ao efeito ano. Mesmo após 20 anos do trabalho pioneiro de Schmalensee, o tema segue atraindo interesse. Diversos trabalhos nessa linha foram publicados em 2003 no Strategic M anagement Journal (Adner e H elfat, 2003; Hawawini, Subramanian e Verdin, 2003; Ruefli eWiggins, 2003; M cNamara, Vaaler e Devers, 2003). Recentemente, uma nova onda de artigos abordou a mesma questão usando os modelos hierárquicos ou multinível (Hough, 2006; Misangyi et al., 2006a; Brito, 2006; Short et al., 2006; Short et al., 2007).

Este trabal ho adota a abordagem multinível, mas explora outro tipo de efeito - 0 efeito país. Analisa uma ampla amostra, com 10.927 firmas de 224 indústrias em 37 diferentes países, num período de 10 anos (1995-2004). 0 efeito país foi apenas recentemente introduzido nos modelos clássicos de análise de componentes da variância (Brito e Vasconcelos, 2003; Makino, Isobe e Chan, 2004; Hawawini, Subramanian e Verdin, 2004; Victer e M CGahan, 2006), não tendo sido ainda abordado na perspectiva de técnicas multinível.

Este artigo traz duas contribuições ao estudo das fontes de heterogeneidade do desempenho das firmas, especificamente no que se refere ao efeito país, derivadas das técnicas multinível. Primeiro, estima, de maneira mais apropriada, a importância relativa dos efeitos país e interação indústria-país, com o uso de uma metodologia mais adequada - que leva em conta a hierarquia natural existente nos dados - e uma amostra selecionada especificamente para analisar esses efeitos. Segundo, vai além de apenas estimar o efeito país na composição da variância do desempenho. Analisa, também, o efeito país para cada um dos países existentes na amostra, que reflete a resultante de todos os fatores específicos àquele país que afetam o desempenho das firmas associadas a ele. Isso tornou possível desenvolver um ranking de países com base na rentabilidade das firmas.

A seção seguinte delineia o referencial teórico que apóia a hipótese da existência de um efeito país sobre o desempenho das firmas e aborda brevemente o resultado de estudos empíricos anteriores sobre o tema. A seguir, são descritos os dados utilizados (uma amostra da Compustat Global) e o método de análise. A próxima seção apresenta os resultados - a dimensão do efeito país e um ranking de países com base na rentabilidade das firmas. Final mente, são discutidas as implicações gerenciais, limitações do trabalho e sugestões para estudos futuros.

\section{FUNDAMENTAÇÃO TEÓRICA}

Os países apresentam diferenças em um amplo número de características que podem influenciar o desempenho das firmas. Dois grandes grupos de tais características dos ambientes nacionais são discutidos nesta seção: as de natureza econômica e institucional.

As diferenças de natureza econômica poderiam ser gradualmente anuladas pelo processo de globalização crescente, mas Ghemawat (2003) oferece argumentos que apóiam a existência de um efeito do país de origem, quando afirma que a economia mundial pode ser melhor caracterizada por um estado de semiglobal ização, marcado pela existência de importantes barreiras à integração econômica dos países, em termos de comércio internacional, distribuição geográfica de FDI, dispersão de preços entre países e fluxo de fatores de produção.

Em linha com as alegações de Ghemawat (2003), Hawawini, Subramanian e Verdin (2004) discutem três razões para a existência de um efeito do país sobre 0 desempenho das firmas. 0 primeiro consiste no viés do país de origem no comércio interno, com uma limitada integração dos mercados de produtos e uma maior dimensão do comércio dentro dos países do que entre países. 0 segundo viés do país de origem refere-se ao financiamento interno e à el evada correlação entre investimento interno e poupança interna, o que sugere que o investimento nacional é primordialmente financiado pela poupança nacional e que o capital parece não cruzar livremente as fronteiras em busca de melhores retornos. 0 terceiro viés do país de origem relaciona-se à 
preferência dos investidores por ações domésticas, com 0 portfólio de investimentos no mercado de capitais fortemente concentrado em empresas domésticas (Hawawini, Subramanian e Verdin, 2004).

Os pontos levantados por Ghemawat (2003) e Hawawini, Subramanian e Verdin (2004) sugerem que as atividades das firmas são fortemente dependentes do ambiente econômico de seu país de origem e que o processo de globalização está longe de tornar irrel evantes as fronteiras nacionais. As diferenças decorrentes do ambiente institucional de cada país (N orth, 1990; Eggertsson, 1990) também podem ter efeito no desempenho das empresas, já que essas diferenças influenciam a construção da base de recursos das firmas e a apropriação das rendas geradas por esses recursos.

O governo - elemento central do ambiente institucional das nações - tem importante papel, como é discutido em termos amplos por Ring et al. (2005) e de maneira mais específica por outros trabalhos, no mesmo número da Academy of $M$ anagement Review. A influência do governo dá-se em diversas facetas das atividades das firmas, entre as quais, na criação de novas indústrias (Spencer, Murthae Lenway, 2005); no processo de inovação e imitação de tecnologias ( $M$ ahmood e Ruffin, 2005); na influência sobre a "atratividade política" dos países e as possibilidades de implementação de estratégias políticas (Bonardi, Hillman e Keim, 2005); e na corrupção (Rodríguez, Uhlenbruck e Eden, 2005).

A construção da base de recursos e capacidades das firmas (sob a óptica da Resource Based View - RBV -, Wernerfelt, 1984; Peteraf, 1993) é afetada diretamente pelo ambiente nacional, como defendido por Wan (2005), ao afirmar que os ambientes dos países apresentam diversos níveis e tipos de fatores e instituições, e que os recursos que as firmas necessitam ou obtêm, e as capacidades subseqüentemente desenvolvidas, são diferentes entre países. Essa perspectiva é coerente com a proposta de Peng (2002) de interação entre instituições, condições da indústria, recursos da firma e ações estratégicas.

A influência do ambiente institucional nacional sobre a construção da base de recursos da firma é, também, enfatizada por Thomas e Waring (1999) no model o environment-conduct-performance. Segundo esses autores, as diferenças entre recursos e estratégias de firmas de distintos países tendem a se manter, já que a dependência de características do ambiente para a acumulação dos recursos agiria como mecanismo de barreira à imitação de concorrentes de outros países (Thomas e Waring, 1999). Esse argumento é consistente com a abordagem de Kogut
(1991), quando enfatiza as diferenças nas capacidades tecnológicas e organizacionais em diferentes nações e destaca que a persistência das diferenças de desempenho entre países seria uma função da permeabilidade relativa das fronteiras da firma em relação às fronteiras do país.

Além de influenciar a construção da base de recursos das firmas, 0 ambiente nacional pode afetar a apropriação pelas firmas das rendas geradas pelos seus recursos. Esse argumento fundamenta-se nas propostas de Foss e Foss (2001), Kim e Mahoney (2005) e Foss e Foss (2005), de integração entre economia de direitos de propriedade, economia de custos de transação (Eggertsson, 1990) e RBV.

Um elemento fundamental dessa perspectiva consiste em que os custos de transação positivos implicam que a maioria dos direitos de propriedade não está totalmente protegida e pode estar sujeita a esforços de captura - atividades que consomem recursos para apropriar-se do valor de outros agentes sem compensá-los por isso. A partir desse conceito de captura, pode-se definir proteção em termos de atividades que consomem recursos para reduzir os incentivos de outros agentes para capturar os direitos de propriedade. Os meios de proteção incluem, entre outros, estruturas de governança que controlam o risco moral e contratos formais (Foss e Foss, 2005).

Os esforços de captura e proteção implicam a dissipação de val or ou a erosão de valor dos recursos (Dierickx e Cool, 1989). Se o detentor de um recurso é capaz de criar e apropriar-se de mais valor de seu feixe de recursos do que os concorrentes, el e tem a possibilidade de obter uma vantagem competitiva sustentada (Foss e Foss, 2005).

Dado que os custos detransação dependem, fundamental mente, dos arcabouços institucionais nacionais ( N orth, 1990; Eggertsson, 1990), firmas com recursos semel hantes em diferentes países podem ter diferentes níveis de dissipação de suas rendas, o que implicaria variações do desempenho financeiro entre países.

Enquanto, sob a óptica da RBV, o ambiente nacional tem implicações sobre a construção da base de recursos das firmas, na perspectiva da organização industrial (Bain, 1956; Porter, 1980) as estruturas industriais podem ser distintas entre países, oferecendo mais ou menos espaço para a obtenção de rendas de monopólio na mesma indústria em diferentes nações, o que também suporta a existência do efeito país.

Final mente, empresas que atuam em determinadas indústrias podem ter um desempenho superior (ou inferior) em determinados países, o que implicaria a existência de um efeito da interação indústria-país. Esse efeito pode ser derivado de diferenças nas estruturas industriais nacio- 


\section{RAFAEL G. BURSTEIN GOLDSZMIDT •LUIZ ARTUR LEDUR BRITO •FLAVIO CARVALHO DE VASCONCELOS}

nais, assim como da configuração do "diamante" nacional (Porter, 1990), de vantagens competitivas oriundas do tipo de sistemas nacionais de negócios (Haake, 2002) e de vantagens comparativas pela variedade de capitalismo do país em questão (Hall e Soskice, 2001).

Essa breve revisão de diferentes abordagens teóricas claramente apóia a hipótese de existência de um efeito do país no desempenho das empresas que nele operam ou que o têm como país de origem. A revisão também indica a possibilidade de um efeito de interação entre o país e a indústria, ou vantagens e desvantagens para combinações específicas de países e indústrias. A próxima seção revisa estudos que tentaram quantificar esses efeitos.

\section{Estudos empíricos anteriores}

U ma das abordagens utilizadas para analisar empiricamente o efeito país no desempenho de empresas foi a da confrontação de resultados de empresas de diferentes países. Alguns dos primeiros estudos que analisaram a relação entre localização e desempenho estavam primordialmente interessados em comparações de firmas japonesas e americanas. Brown, Soybel e Stickney (1994) compararam o desempenho de firmas japonesas e norte-americanas em 11 indústrias no período de 1985 a 1988, e não encontraram diferenças entre ambas em termos de margens de lucro. As firmas norte-americanas, porém, tiveram maior giro dos ativos e, conseqüentemente, maior retorno sobre ativos. Brouthers (1998), em estudo análogo, comparou o desempenho de 167 firmas de manufatura norteamericanas e japonesas e obteve resultados semel hantes, concluindo que as primeiras apresentavam desempenho significativamente superior em termos de retorno sobre ativos (ROA).

Collins (1990) comparou o desempenho de 133 empresas listadas pela US Fortune 500 e concluiu que aquelas com atividades exercidas preponderantemente em países desenvolvidos tinham desempenho superior àquelas concentradas em países em desenvolvimento. 0 risco, mensurado como o desvio padrão das taxas de retorno mensais, foi significativamente menor para as firmas diversificadas em países em desenvolvimento.

Resultados distintos dos de Collins (1990) foram obtidos por Makino, Beamish e Zhao (2004), que analisaram dados de 26.857 investimentos estrangeiros diretos de empresas japonesas em 150 países, no período de 1991 a 1999. A rentabilidade foi maior nos países menos desenvolvidos, que apresentaram, também, maior variabilidade do desempenho. Esses resultados sugerem que operar em países desenvolvidos torna o desempenho das subsidiárias mais estáveis. Por outro lado, o desempenho médio mais el evado nos países pouco desenvolvidos vem acompanhado por um maior risco, expresso pela variabilidade do retorno.

Um conjunto mais amplo de variáveis explicativas foi considerado por Christman, Day e Yip (1999). A partir de dados de 99 subsidiárias de quatro MNCs do ramo de consumer packaged goods - duas norte-americanas e duas européias, distribuídas em 37 diferentes países, no período de 1980 a 1984 - , os autores anal isaram o efeito de várias características dos países de operação das subsidiárias ( nível de desenvolvimento - dummy, tamanho da população, taxa de inflação e estabilidade política), das M N Cs, das subsidiárias e da indústria sobre as margens brutas de cada subsidiária. A escolha do ramo de negócios deu-se em função da maior autonomia estratégica que suas subsidiárias tendem a apresentar. Um modelo de regressão múltipla explicou $74 \%$ da variância do desempenho, e as características do país responderam por $39 \%$. Nos países menos desenvolvidos, o desempenho foi inferior.

Vistos em conjunto, esses estudos comprovam, empiricamente, diferenças de desempenho das empresas entre diferentes países e, em alguns casos, explicam essas variações com variáveis econômicas.

Recentemente, model os de análise de componentes da variância têm sido utilizados para analisar o efeito país oferecendo uma perspectiva mais general ista da variabilidade provocada por esse efeito frente às demais fontes de variação do desempenho. Furman (2000) decompôs a variância do desempenho de 763 firmas da Austrália, 1.264 do Canadá, 7.048 da Inglaterra e 13.272 originárias dos Estados Unidos, a partir de dados da base Worldscope do período 1992-1996. Um modelo de ANOVA mostrou fortes diferenças entre a dimensão dos efeitos em cada país, com o efeito firma de $51 \%$ na Austrália, 18,1\% na Inglaterra e 9,4\% no Canadá. Tais diferenças foram interpretadas por Furman como resultado do efeito do país. As composições de indústrias em cada país, no entanto, foram muito diferentes, e o número de casos para comparação em um mesmo setor era muito pequeno, limitando a confiabilidade dos resultados.

Avançando um passo além de Furman (2000), Brito e Vasconcel os (2003) - com dados da Compustat Global de empresas, em 78 países - incluíram diretamente o efeito país e o efeito interação indústria-país em modelos de análise de componentes da variância. 0 efeito país mostrou-se relevante ao explicar uma parcela importante da variância total, da mesma ordem de grandeza da indústria, e variou bastante por divisão da Standard Industrial Classification, ou SIC (Classificação Industrial Padrão). 0 
país explicou até $17,7 \%$ da variância do desempenho na agricultura, 13,5\% em construção, enquanto a interação país-indústria foi responsável por $11,7 \%$ em construção e $45 \%$ em transportes.

A partir da mesma base de dados (Compustat G lobal), Victer e McGahan (2006) utilizaram a ANOVA e não incluíram o efeito firma, mas apenas indústria, país de origem, ano e suas interações. 0 maior efeito foi 0 da interação país-indústria, que respondeu por 14,79\% da variância total. No que se refere apenas à divisão de manufatura, a fração da variância explicada pelo modelo foi maior, assim como os efeitos relativos ao país (2,35\%), país-ano (5,13\%) e país-indústria (16,51\%). É importante destacar que, na ausência do efeito firma, a variância entre empresas é captada, em parte, por outros efeitos, 0 que implica seu incremento. Isso pode ter ocorrido, particularmente, no efeito da interação indústria-país, já que o processo de tratamento da amostra por Victer e M cGahan não se preocupou com o número de firmas existente em cada interação.

Lançando mão de outra base de dados e diferentes indicadores de desempenho, Hawawini, Subramanian e Verdin (2004) estudaram o efeito país de origem com base em dados de desempenho de 1.305 firmas de manufatura e serviços de seis países, três grandes economias abertas (EUA, Reino Unido, Alemanha) e três pequenas economias abertas (Holanda, Bélgica e Luxemburgo), entre 1993 e 1996. Utilizaram os indicadores de desempenho lucro econômico sobre capital empregado (EP/CE) evalor total de mercado sobre capital empregado (TMV/CE), os quais refletem desempenho econômico e não contábil, além de não serem afetados por convenções contábeis que distorcem indicadores, tais como ROA.

Modelos de análise de componentes de variância indicaram a predominância do efeito firma, um efeito país de $0,2 \%$ para lucro econômico sobre capital empregado e $0,7 \%$ para valor total de mercado sobre capital empregado. 0 efeito da interação indústria-país foi de 2,1 e 1,0\%, respectivamente, e a interação indústria-ano, 0,3 e 0\%, com erro de $73 \%$ e $60,7 \%$. 0 efeito firma individual foi o predominante, com $23,8 \%$ para EP/CE e $32,7 \%$ para TMV/CE. Os próprios autores admitem, no entanto, que o reduzido efeito país pode derivar da intensa integração econômica entre os países considerados na amostra (Hawawini, Subramanian e Verdin, 2004). Assim como em Furman (2002), a composição da variância foi muito distinta entre os países, com um efeito firma de $40,7 \%$ para a Inglaterra, 20,6\% para a Alemanha e efeito nulo para Bél gica, Holanda e Luxemburgo.

A consistência da dimensão do efeito país em diferen- tes regiões geográficas foi o interesse central de Brito e Vasconcelos (2004), que analisaram a variância de 462 firmas da América Latina e encontraram um efeito firma muito menor que em outros estudos e el evados efeitos país, ano e interação país-ano. Brito e Vasconcelos (2003), Hawawini, Subramanian e Verdin (2004) e Victer e M cGahan (2006) anal isaram, primordialmente, o efeito país de origem, já que utilizaram dados agregados que não permitem identificar o desempenho das subsidiárias nacionais.

Já M akino, Isobee Chan (2004) estudaram a composição da variância do desempenho de 5.183 subsidiárias de 616 multinacionais japonesas, no período 1996 a 2001. Identificaram um efeito país anfitrião com 4,3\% da variância total, quase tão el evado quanto o efeito indústria, de 5,0\%, e uma interação país-ramo de negócios que explicava 7,5\% da variância total. Mais além, o efeito país mostrou-se mais rel evante em países em desenvolvimento do que em países desenvolvidos.

Em termos gerais, os resultados acerca do efeito país são muito variados entre si. As estimativas de sua magnitude variaram de $0,2 \%$, em Hawawini, Subramanian e Verdin (2004) e Brito e Vasconcelos (2003) para a Divisão de Transportes; a 17,7 \% para a Divisão de Agricultura e Mineração em Brito e Vasconcelos (2003); enquanto a soma dos efeitos país e interação indústria-país esteve entre $2 \%$, em Hawawini, Subramanian e Verdin (2004); $22 \%$, em Victer e M cGahan (2006); e 45\% para a Divisão de Transportes, em Brito e Vasconcel os (2003). Essas diferenças entre os resultados estão provavel mente associadas à diversidade de critérios para sel eção da amostra e métodos de análise. Por outro lado, os estudos de componentes de variância limitaram-se a estimar o percentual da variância associado ao país ou suas interações e não foram capazes de efetivamente comparar esse efeito entre países. N este estudo, a extensão da amostra, o processo de sua seleção e o uso do método multinível oferecem contribuições que minoram essas limitações.

\section{METODOLOGIA}

\section{Operacionalização do desempenho}

0 desempenho foi operacionalizado, neste trabal ho, como o lucro líquido sobre ativos (Return On Assets - ROA), de acordo com a maioria dos estudos anteriores sobre a composição da variância do desempenho. Embora haja importantes limitações de ROA - dado que reflete apenas o lucro contábil -, os resultados com outros indicadores de desempenho baseados no lucro econômico ou no 
valor das firmas no mercado de capitais têm sido muito semel hantes aos obtidos com esse método (Hawawini, Subramanian e Verdin, 2003).

\section{Fonte de dados e tratamento da amostra}

A base de dados Compustat Global (Standard \& Poor's, 2006) foi a fonte de dados para a pesquisa. Os dados foram extraídos de todos os conjuntos de firmas disponíveis na base no período de 1995 a 2004, exceto por Financial Research. A base original contava 23.334 firmas em 87 países e 458 indústrias (definidas pela SIC de quatro dígitos), num período de 10 anos (1995-2004), totalizando 233.340 observações. Esse conjunto de dados foi tratado de acordo com diversos critérios.

Em primeiro lugar, foram eliminadas 2.240 observações vinculadas a firmas sem indicação de SIC ou com SIC iniciada por 9, que inclui estabelecimentos não-classificáveis e governo. Outras 26.810 observações de empresas financeiras (SIC 6.000) foram excluídas, já que seus indicadores de desempenho não são diretamente comparáveis às demais indústrias. Tal como proposto por McGahan e Porter (1997), foram desconsideradas as firmas com vendas médias no período inferiores aUS\$ 10 milhões ( 20.800 observações), ou ativos médios inferiores ao mesmo valor (2.540 observações). Em 45.024 observações, a variável dependente não se encontrava na base, o que implicou sua eliminação da análise.

Os critérios seguintes buscaram evitar a indeterminação na al ocação dos diferentes efeitos pela presença de menos de três casos em cada fator ou interação de fatores analisados. M antiveram-se apenas as firmas que apresentaram três ou mais anos da variável dependente relatados na base, o que levou à exclusão de 2.060 observações. Em 193 observações, havia menos de três firmas em uma mesma SIC num dado ano e, em 277, menos de três firmas num dado país em um determinado ano.

Como o efeito da interação indústria-país inclui-se na análise, eliminaram-se, também, 37.237 observações em interações indústria-país que, em determinado ano, contaram menos de três firmas. Mais 254 observações de países que contavam menos de três indústrias foram excluídas, assim como 11.418 observações de indústrias que se encontravam em menos de três países. Este último número merece um comentário à parte. Dado que a Compustat Global apresenta uma elevada fração defirmas norte-americanas e japonesas, e o critério de definição da indústria é bastante específico (SIC de quatro dígitos), em muitos casos havia indústrias com firmas apenas nesses dois países, o que explica o alto número de observações eliminadas por essa razão.
Finalmente, 846 observações $(0,4 \%$ do total) apresentavam resultado operacional sobre ativos superior a $100 \%$ ou inferior a $-100 \%$. Em função de considerações devalidade do construto e possíveis erros nos dados, essas observações foram eliminadas. Ao final da depuração, a base contava 83.641 observações e 10.927 firmas, ou $42 \%$ da base original, após a exclusão das firmas sem SIC e das que atuam no ramo financeiro.

\section{Método de Análise}

Os dados de desempenho de firmas - observações anuais de diferentes firmas, em distintas indústrias ou países têm uma natureza hierárquica, ou seja, estão organizados em diferentes níveis de agregação. Os dados de desempenho de firmas, além de hierárquicos, caracterizam-se como de medidas repetidas, ou seja, o valor da variável de interesse é mensurado, para um mesmo caso (firma), em diferentes momentos (anos).

Misangyi et al. (2006b) comparam as técnicas de ANOVA de medidas repetidas, ANOVA multivariada de medidas repetidas e model os multinível em dados hierárquicos longitudinais. Concluem que os modelos multinível são preferíveis particularmente quando a premissa de esfericidade não é respeitada, quando faltam dados ou quando os dados não são balanceados (número de casos diferentes entre grupos), situações encontradas no que se refere a desempenho de firmas.

Outra característica relevante dos modelos multinível é a possibilidade de model ar efeitos em que há classificação cruzada no mesmo nível (Raudenbush e Bryk, 2002; Snijders eBosker, 1999), por exemplo, indústrias e países. Meyers (2004) analisa o impacto do tratamento inadequado de classificações cruzadas em modelos multinível e conclui que, entre outras conseqüências, as estimativas de componentes de variância são enviesadas.

\section{A estimação da importância relativa do efeito país}

Um modelo multinível nulo (sem variáveis explicativas) pode ser utilizado para decompor a variância da variável dependente (de maneira análoga à ANOVA ou à análise de componentes da variância), levando em conta, porém, a estrutura hierárquica dos dados.

Dois efeitos consistem no interesse central deste trabalho: país e interação indústria-país, além dos efeitos indústria e firma, que também serão anal isados. Os efeitos país e indústria devem ser considerados como de classificação cruzada no mesmo nível, já que os países não estão aninhados em indústrias e tampouco as indústrias estão aninhadas em países. Assim, o nível mais alto deve incluir a classificação cruzada de indústrias e 
países (efeitos principais), assim como a interação indústria-país. As firmas estão aninhadas em interações indústria-país e, final mente, as observações anuais, aninhadas em firmas.

Assim, a estimação dos efeitos país e interação indústria-país requer um modelo de três níveis, com países (I), indústrias (k) e interações indústria-país (kl) no nível 3; firmas (j) no nível 2; e os anos (i) no nível 1. Em termos formais:

$$
\text { Nível } 1-R O A_{i j k l}=\pi_{0 j k l}+e_{i j k l} \quad e_{i j k l} \sim N\left(0, \sigma_{e}^{2}\right)
$$

em que $\pi_{0 \mathrm{jkl}}$ é o desempenho médio da firma j e $\mathrm{e}_{\mathrm{ijkl}}$ é 0 desvio do desempenho médio dessa firma no ano $\mathrm{i}$.

$$
\text { Nível } 2-\pi_{0 j k l}=\beta_{00 k l}+r_{0 j k l} \quad r_{0 j k l} \sim N\left(0, \sigma_{r}^{2}\right)
$$

em que $\beta_{00 k 1}$ é o desempenho médio das firmas da indústria k no país I e $r_{0 j k l}$ é o desvio do desempenho da firma j em relação a essa média.

$$
\begin{array}{ll}
\text { Nível 3- } \beta_{00 k l}=\gamma_{0000}+s_{000 k}+t_{000 l}+u_{00 k l} & s_{000 k} \sim N\left(0, \sigma_{s}^{2}\right) \\
& t_{000 l} \sim N\left(0, \sigma_{t}^{2}\right) \\
& u_{00 k l} \sim N\left(0, \sigma_{u}^{2}\right)
\end{array}
$$

em que $\gamma_{0000}$ é o desempenho médio de toda a amostra, s000k é o resíduo da indústria $k$ em relação a essa média (efeito indústria), $\mathrm{t}_{0001}$ é o resíduo do país I em relação à esta média (efeito país) e u $u_{00 k \mathrm{l}}$ é o resíduo da interação indústria k - país I (efeito da interação indústria-país). Em notação compacta:

$$
R O A_{i j k l}=\gamma_{0000}+s_{000 k}+t_{000 l}+u_{00 k l}+r_{0 j k l}+e_{i j k l} \quad \text { Eq. } 1
$$

Quanto à interpretação da fração da variância explicada por cada efeito, Brush e Bromiley (1997), com base em um estudo de simulação, mostram que "a importância de um efeito é aproximadamente a raiz quadrada do componente de variância" (Brush e Bromiley, 1997, p. 833). Considerando-se esse argumento, são analisados tanto os componentes da variância quanto à sua raiz quadrada, denominando-se o segundo, no presente trabalho, "importância relativa" dos efeitos.

\section{0 efeito país específico}

Um importante uso prático dos modelos multinível consiste em monitorar o desempenho de organizações individuais - firmas, escolas ou sal as de aula (Raudenbush e Willms, 1995). Model os hierárquicos de dois níveis (es- cola e alunos) têm sido amplamente usados para a análise do desempenho de escolas. No nível 1 são incluídas variáveis independentes que caracterizam os alunos, tais como o contexto familiar e habilidades prévias e al gum indicador de desempenho escolar como variável dependente. A diferença entre o desempenho médio previsto, dadas as características dos alunos, e o seu desempenho observado é atribuída à escola. Um resíduo significante no nível 2 (escola) indicaria a existência de um efeito da escola em questão.

Este trabalho propõe a utilização da estratégia delineada acima para avaliar o efeito país específico sobre o desempenho das firmas. Denomina-se efeito país específico à diferença entre o valor esperado do desempenho das firmas em um país, dada a sua composição de indústrias e seu desempenho observado.

Vale ressaltar a diferença conceitual entre o efeito país e o efeito país específico. Considerando-se o modelo formalizado na Eq. 1, os resíduos do nível 3 relativos ao país (t000l) têm distribuição normal e variância $\sigma_{t}^{2}$. 0 efeito país consiste na relação entre essa variância e a variância total (também denominada correlação intraclasse). Já quanto ao efeito país específico, o objetivo é a prediçãol de t000l, o resíduo associado ao país I, para cada país individualmente.

Esses resíduos indicam o efeito médio de cada país sobre a rentabilidade das firmas. Como o modelo inclui a classificação cruzada por indústria, o efeito de diferentes composições industriais entre países é controlado. A partir desses resíduos é possível el aborar um ranking de efeitos país específico sobre o desempenho das firmas, ou da medida em que, em determinados países, as firmas obtêm uma rentabilidade superior (ou inferior).

A alternativa escolhida neste trabal ho para predição de $_{\text {0001 }}$ consiste no estimador empírico bayesiano. Ele se baseia em dois tipos de informação: os dados do país I e a premissa do modelo de que a variável $t_{0001}$ apresenta distribuição normal, com média 0 e variância igual a $\sigma_{t}^{2}$. Esses estimadores apresentam a característica de encol himento (shrinkage), ou seja, são enviesados em direção à média. Países com amostras menores tendem a apresentar estimativas instáveis de $t_{0001 .}$ Assim, quanto menor o número de observações no país e mais el evada a variância do desempenho dentro do país, maior o encolhimento do estimador empírico bayesiano e mais elevado o seu erro padrão (Raudenbush e Bryk, 2002).

Esse viés de encol himento, no entanto, implica previsões mais eficientes, com menores erros padrão e, conseqüentemente, intervalos de previsão mais estreitos 
(Snijders e Bosker, 1999). As previsões são, também, mais adequadas quando o número de casos no nível inferior (firma) por unidade de nível superior (país) é pequeno (Raudenbush e Bryk, 2002).

Nos países em que o resíduo for significante, há evidências para afirmar que existe um efeito país. A existência desse efeito, no entanto, não permite inferências causais acerca do impacto de características do ambiente nacional sobre o desempenho das firmas, mas apenas uma constatação descritiva, dada a ausência de variáveis de controle e todas as limitações associadas a desenhos de pesquisa quase-experimentais e não experimentais (Pedhazur e Schmelkin, 1991).

\section{RESULTADOS E DISCUSSÃO}

Dos 87 países inicialmente presentes na base da Compustat Global, restaram 37 após o tratamento da base de dados (África do Sul, Alemanha, Argentina, Austrália, Áustria, Bélgica, Bermudas, Brasil, Canadá, Chile, China, Coréia do Sul, Dinamarca, Espanha, EUA, Finlândia, França, Grécia, Holanda, Hong Kong, Ilhas Caimãs, Índia, Indonésia, Israel, Itália, Japão, Malásia, M éxico, N oruega, N ova Zelândia, Reino Unido, Rússia, Cingapura, Suécia, Suíça, Tailândia e Taiwan).

Os demais 50 países, em conjunto, representavam uma pequena fração da base inicial. Apesar desta redução no número de nações, há, ainda, representantes de todos os continentes. 0 conjunto dos 37 países inclui todas as 20 maiores economias e representa mais de $86 \%$ do PIB mundial, o que oferece indícios de que os dados, em termos de distribuição geográfica, refletem a atividade econômica global.

Há uma concentração das observações nos Estados Unidos, Japão e China, que respondem, em conjunto, por mais de $60 \%$ da base. No entanto, duas razões tornam tal concentração uma preocupação menor. Em primeiro lugar, esses países são responsáveis por mais de $40 \%$ do PIB mundial. Em segundo lugar, os modelos multinível são robustos quando utilizados nesse tipo de composição não balanceada da amostra ( M isangyi et al., 2006b), sendo essa uma das vantagens metodológicas deste trabal ho frente aos métodos tradicionais de componentes de variância.

\section{Os efeitos país e indústria-país}

Os resultados do model o formalizado na Eq.1 (Tabela 1) são relatados em termos de composição da variância e sua raiz quadrada, que reflete de maneira mais adequada a importância relativa de cada efeito (Brush e Bromiley, 1997). As frações de variância associadas aos efeitos país (de 2,1 a 3,6\%), indústria (1,2 a 2,9\%) e indústria-país (de 2,9 a 3,5\%) são significantes. Suas magnitudes são semelhantes quando analisadas toda a amostra, apenas a divisão de Manufatura e as demais divisões da SIC.

Tabela 1 - Composição da variância e importância relativa dos efeitos.

\begin{tabular}{|c|c|c|c|c|c|c|c|c|c|}
\hline & \multicolumn{3}{|c|}{ AM OSTRA COM PLETA } & \multicolumn{3}{|c|}{ MANUFATURA } & \multicolumn{3}{|c|}{$\begin{array}{l}\text { DEMAIS DIVISÕES DA SIC } \\
\text { (EXCETO MANUFATURA) }\end{array}$} \\
\hline & VAR. & $\%$ VAR. & $\begin{array}{l}\text { IMPORT. } \\
\text { RELATIVA }\end{array}$ & VAR. & $\%$ VAR. & $\begin{array}{l}\text { IMPORT. } \\
\text { RELATIVA }\end{array}$ & VAR. & $\%$ VAR. & $\begin{array}{l}\text { IMPORT. } \\
\text { RELATIVA }\end{array}$ \\
\hline País & 5,58 & $3,2 \%$ & $9,7 \%$ & 2,92 & $2,1 \%$ & $8,1 \%$ & 7,42 & $3,6 \%$ & $10,1 \%$ \\
\hline Indústria & 4,44 & $2,5 \%$ & $8,6 \%$ & 1,69 & $1,2 \%$ & $6,2 \%$ & 5,96 & $2,9 \%$ & $9,0 \%$ \\
\hline Indústria-país & 5,03 & $2,9 \%$ & $9,2 \%$ & 4,38 & $3,1 \%$ & $9,9 \%$ & 7,24 & $3,5 \%$ & $10,0 \%$ \\
\hline Firma & 57,03 & $32,7 \%$ & $31,0 \%$ & 47,04 & $33,5 \%$ & $32,4 \%$ & 65,69 & $31,6 \%$ & $30,0 \%$ \\
\hline Tempo* & 102,54 & $58,7 \%$ & $41,5 \%$ & 84,23 & $60,1 \%$ & $43,4 \%$ & 121,62 & $58,5 \%$ & $40,9 \%$ \\
\hline Total & 174,62 & $100,0 \%$ & $100,0 \%$ & 140,26 & $100,0 \%$ & $100,0 \%$ & 207,93 & $100,0 \%$ & $100,0 \%$ \\
\hline Países & \multicolumn{3}{|c|}{37} & \multicolumn{3}{|c|}{35} & \multicolumn{3}{|c|}{37} \\
\hline Indústrias & \multicolumn{3}{|c|}{224} & \multicolumn{3}{|c|}{124} & \multicolumn{3}{|c|}{100} \\
\hline Firmas & \multicolumn{3}{|c|}{10,927} & \multicolumn{3}{|c|}{5,318} & \multicolumn{3}{|c|}{5,609} \\
\hline
\end{tabular}

Fonte: Análise dos autores a partir da Compustat Global.

* O efeito tempo é análogo à "variância não explicada" nos modelos de análise de componentes da variância. 
Em termos de importância relativa (indicada pela raiz quadrada dos componentes da variância), há dois grupos de efeitos aproximadamente da mesma magnitude - efeitos do ambiente externo (país, indústria e indústria-país, que somam $27,5 \%$ para toda a amostra) e efeito firma (31,0\%). A importância relativa dos efeitos foi também estimada para dois outros indicadores de desempenho Resultado Operacional sobre Ativos (que exclui juros e impostos) e Resultado O peracional sobreVendas (que não é influenciado pelo giro do ativo total e seria mais adequado para comparações internacionais - de acordo com Makino, Isobe e Chan, 2004). Os resultados são muito semel hantes para os três indicadores, o que reforça a validade dos resultados.

Finalmente, a estabilidade do efeito país ao longo do tempo foi analisada por meio de uma abordagem semeIhante à adotada por M cN amara, Valeers, Devers (1994), a qual se baseia na divisão da amostra em janelas de quatro anos. A decomposição da variância do desempenho em cada janela permitiu concluir que os efeitos país e interação indústria-país se mantêm relativamente estáveis no período de análise.

Os resultados obtidos com os modelos multinível e os modelos de análise de componentes de variância foram muito semel hantes. Isso é consistente com os resultados de Hough (2006), que compara os resultados obtidos com a decomposição da variância por meio de análise de componentes da variância e model os multinível e encontra diferenças, embora pouco significativas. A principal contribuição dos modelos multinível, porém, não consiste em estimar de maneira mais adequada a composição da variância do desempenho, mas sim em permitir outras possibilidades, como a análise da influência de países específicos sobre o desempenho das firmas.

A Tabela 2 mostra o efeito país específico previsto para toda a amostra, no período 1995-2004, na primeira metade do período analisado (1995-1999) e na metade final (2000-2004). 0 valor do efeito representa, em pontos percentuais, a diferença entre o ROA médio observado e o ROA esperado, dada a composição de indústrias do país.

Holanda, África do Sul, China, Taiwan e Reino Unido têm efeitos positivos e significantes, enquanto Japão, Malásia, EUA, Canadá, Alemanha, Bermudas, Ilhas Caimãs, Noruega, Suécia e Indonésia apresentam efeitos negativos e significantes. A posição dos países não é estática ao longo do tempo. A comparação dos rankings nas duas janelas de tempo analisadas mostra mudanças importantes. 0 Brasil, por exemplo, passa de um efeito negativo, na 36a posição em 1995-1999, para a 5a posição, com efeito positivo em 2000-2004.

Tabela 2 - Efeito país específico.

\begin{tabular}{|c|c|c|c|c|c|c|}
\hline \multirow[b]{2}{*}{ PAÍS } & \multicolumn{2}{|c|}{ AMOSTRA COMPLETA } & \multicolumn{2}{|c|}{ 1995-1999 } & \multicolumn{2}{|c|}{$2000-2004$} \\
\hline & $\begin{array}{l}\text { EFEITO PAÍS } \\
\text { ESPECÍFICO }\end{array}$ & RANKING & $\begin{array}{l}\text { EFEITO PAÍS } \\
\text { ESPECÍFICO }\end{array}$ & RANKING & $\begin{array}{l}\text { EFEITO PAÍS } \\
\text { ESPECÍFICO }\end{array}$ & RANKING \\
\hline Holanda & $3,61 *$ & 1 & $4,47^{*}$ & 1 & 1,72 & 8 \\
\hline África do Sul & $3,14 *$ & 2 & 2,51 & 4 & 2,98 & 2 \\
\hline China & $2,77^{*}$ & 3 & $3,86^{*}$ & 2 & $1,54^{*}$ & 12 \\
\hline Taiwan & $2,52 *$ & 4 & 0,60 & 15 & $3,25^{*}$ & 1 \\
\hline Grécia & 2,38 & 5 & 1,52 & 7 & 1,93 & 6 \\
\hline Espanha & 1,88 & 6 & 1,26 & 10 & 2,29 & 4 \\
\hline Nova Zelândia & 1,85 & 7 & 1,57 & 6 & 1,19 & 14 \\
\hline Índia & 1,66 & 8 & 0,93 & 12 & 1,79 & 7 \\
\hline México & 1,51 & 9 & 1,33 & 9 & 1,44 & 13 \\
\hline Finlândia & 1,36 & 10 & 2,34 & 5 & 1,13 & 15 \\
\hline Rússia & 1,31 & 11 & $-0,62$ & 25 & 2,57 & 3 \\
\hline Reino Unido & $1,27 *$ & 12 & $1,39 *$ & 8 & $-0,19$ & 22 \\
\hline
\end{tabular}


RAFAEL G. BURSTEIN GOLDSZMIDT •LUIZ ARTUR LEDUR BRITO •FLAVIO CARVALHO DE VASCONCELOS

(continuação)

\begin{tabular}{|c|c|c|c|c|c|c|}
\hline Dinamarca & 1,16 & 13 & $-0,36$ & 21 & 1,09 & 16 \\
\hline Coréia & 1,05 & 14 & $-0,96$ & 27 & $1,65^{*}$ & 11 \\
\hline Chile & 1,01 & 15 & 0,74 & 13 & 0,97 & 17 \\
\hline Israel & 0,82 & 16 & 0,57 & 16 & 0,02 & 20 \\
\hline Austrália & 0,77 & 17 & $-0,39$ & 22 & $1,69 *$ & 9 \\
\hline Tailândia & 0,54 & 18 & $-0,85$ & 26 & $1,68^{*}$ & 10 \\
\hline Hong Kong (China) & 0,29 & 19 & $-0,43$ & 23 & 0,35 & 18 \\
\hline Suíça & 0,00 & 20 & 0,16 & 20 & $-0,45$ & 25 \\
\hline França & $-0,08$ & 21 & 0,21 & 19 & $-0,19$ & 23 \\
\hline Bélgica & $-0,16$ & 22 & 1,06 & 11 & $-0,04$ & 21 \\
\hline Cingapura & $-0,18$ & 23 & 0,39 & 17 & 0,04 & 19 \\
\hline Argentina & $-0,41$ & 24 & 0,31 & 18 & $-0,61$ & 26 \\
\hline Áustria & $-0,98$ & 25 & 0,63 & 14 & $-1,34$ & 28 \\
\hline Japão & $-1,03 *$ & 26 & $-2,09 *$ & 32 & $-0,37$ & 24 \\
\hline Malásia & $-1,26^{*}$ & 27 & $-1,29 *$ & 28 & $-1,21 *$ & 27 \\
\hline Brasil & $-1,33$ & 28 & $-4,05^{*}$ & 36 & 2,00 & 5 \\
\hline EUA & $-1,35^{*}$ & 29 & $-1,75^{*}$ & 31 & $-1,66^{*}$ & 29 \\
\hline Canadá & $-1,38 *$ & 30 & $-2,14 *$ & 33 & $-1,84 *$ & 31 \\
\hline Itália & $-1,66$ & 31 & $-0,61$ & 24 & $-1,84$ & 30 \\
\hline Alemanha & $-2,06 *$ & 32 & $-1,38 *$ & 29 & $-2,18 *$ & 32 \\
\hline Bermudas & $-3,04 *$ & 33 & $-2,54^{*}$ & 34 & $-2,89 *$ & 33 \\
\hline Ilhas Caimãs & $-3,41^{*}$ & 34 & 2,90 & 3 & $-3,15^{*}$ & 34 \\
\hline Noruega & $-3,47 *$ & 35 & $-1,62$ & 30 & $-4,32 *$ & 36 \\
\hline Suécia & $-3,58 *$ & 36 & $-3,03 *$ & 35 & $-4,39 *$ & 37 \\
\hline Indonésia & $-3,66 *$ & 37 & $-4,23 *$ & 37 & $-3,26 *$ & 35 \\
\hline
\end{tabular}

Fonte: Análise dos autores a partir da Compustat Global.

* Indica que o efeito país específico é significante a $5 \%$.

Em termos gerais, países emergentes, como China e Taiwan, estão em destaque, enquanto as maiores economias mundiais - Estados Unidos, Japão e Alemanha encontram-se em posições muito inferiores. Esse ranking parece estar invertido em rel ação a outras classificações de países, como as baseadas nos índices de competitividade das nações desenvolvidos pelo Institute for M anagement Development (Garelli, 2006) e World Economic Forum (Porter, 2004), fortemente correlacionad os com o PIB per capita, em que os países emergentes se colocam muito abaixo das grandes economias.
0 ranking do presente trabalho é conceitualmente muito distinto desses rankings de competitividade das nações. Os últimos destacam a manutenção e o aumento da renda nacional real como o objetivo final da competitividade, refletido no padrão de vida do país. Essa abordagem coloca a competitividade da nação como um meio para o desenvolvimento econômico. Traz, ainda, um foco no comércio internacional em termos de participação no mercado, balança comercial, investimento e outros indicadores semel hantes (Waheeduzzamman e Ryans jr., 1996), e não a lucratividade das firmas. 
Se a estratégia pode ser conceitual izada em termos de exploração de imperfeições de mercado (Porter, 1980), pode-se esperar que países com arcabouços institucionais mais desenvolvidos e com mecanismos de mercado mais eficientes ofereçam menos espaço para a exploração de imperfeições e a obtenção de lucros extraordinários (Bain, 1956). Sob essa perspectiva, pode-se esperar uma relação inversa entre os indicadores de competitividade das nações e os índices de lucratividade das firmas. Ou seja, ambientes com mais imperfeições de mercado seriam propícios para a obtenção de lucro econômico pelas firmas, embora implicassem limitações em termos de competitividade das nações e desenvolvimento econômico.

\section{CONCLUSÕES E IMPLICAÇÕES}

Este trabalho buscou identificar a importância relativa do efeito país e sua influência em países específicos. A decomposição da variância por meio de modelos lineares hierárquicos com classificação cruzada mostrou efeitos país e interação indústria-país significantes e significativos. A dimensão do efeito país foi semel hante à do efeito indústria, e os efeitos relativos ao ambiente externo (país, indústria-país e indústria) mostraram-se, em conjunto, tão importantes quanto o efeito firma. Apesar da tendência de integração dos mercados nacionais, o efeito país manteve-se estável nos últimos dez anos.

Além de estimar a importância relativa do efeito país, foi possível, com o uso da metodologia multinível, quantificar o efeito de cada país sobre o desempenho das suas firmas (efeito país específico). Utilizando-se essa estimativa do efeito de cada país, foi elaborado um ranking de países, que apresentou posições, em termos gerais, invertidas em relação aos rankings de competitividade das nações. Estes últimos, na maioria das vezes, não estão baseados no desempenho das firmas, mas sim em aval iações de aspectos estruturais da economia que favoreceriam o desenvolvimento. 0 efeito desses fatores na rentabilidade das firmas, como foi avaliado neste trabalho, pode ser bastante diferente.

Por exemplo, o tempo necessário para abrir uma empresa é normal mente usado para estimar o nível de burocracia de determinada economia, e considera-se que os países nos quais os tempos são menores são mais competitivos. Neste trabal ho, não estamos fazendo um jul gamento sobre o que é melhor ou pior, mas apenas aval iando o impacto resultante do país na rentabilidade das firmas. É possível que um maior tempo para a abertura de uma empresa seja prejudicial para o desenvolvimento econômico, mas seja benéfico para a rentabilidade das firmas já existentes! Outro aspecto relevante foi que as posições de al guns países não se mostraram estáveis ao longo do período de análise, com uma significativa mudança do efeito país específico ao longo do tempo.

Os resultados apresentados possuem importantes limitações. A amostra não é probabilística, e, portanto, a análise é frágil em termos de validade externa. Todos os indicadores de desempenho são de natureza contábil e desconsideram o custo de capital, que varia significativamente entre países. A consistência dos resultados para três diferentes indicadores de desempenho contábil, no entanto, reforça a vali dade dos achados. O utra importante limitação refere-se à base de dados utilizada (Compustat Global), que agrega os resultados de firmas multinacionais, o que implica a impossibilidade de separar os efeitos país de origem e país anfitrião. Uma base de dados de desempenho de subsidiárias locais (análoga à utilizada por Makino, Isobe e Chan, 2004), pertencentes, porém, a firmas de diferentes nacionalidades, permitiria essa análise separada.

O principal desafio que se coloca a partir dos achados deste trabal ho consiste em explicar por que as firmas de al guns países são mais lucrativas do que as de outros, eque fatores impulsionam a mudança do efeito país específico ao longo do tempo. Nesse sentido, o modelo utilizado para decompor a variância do desempenho poderia incluir, em estudos futuros - no nível 3 - , variáveis que caracterizam os ambientes nacionais, entre as quais índices de competitividade das nações. Quanto à dinâmica temporal do desempenho, a análise de curvas de crescimento (Singer e Willet, 2003) é um caminho promissor.

Finalmente, há um vasto campo para análise da relação entre desenvolvimento econômico, competitividade das nações e desempenho das firmas. Em termos de Administração de Empresas, o estudo dessa relação pode influenciar as decisões de investimento e estratégias internacionais. Quanto ao campo da Administração Pública, ele pode oferecer subsídios para a análise de impacto de políticas industriais em termos de quem colhe os seus frutos - o país, com desenvolvimento econômico, ou as firmas, com aumento de lucratividade?

\section{NOTA}

$1 \mathrm{Como} \mathrm{t}_{\text {00ol }}$ é uma variável aleatória enão um parâmetro, o termo adequado é predizer e não estimar (SNIJDERS, BOSKER, 1999). 


\section{RAFAEL G. BURSTEIN GOLDSZMIDT •LUIZ ARTUR LEDUR BRITO •FLAVIO CARVALHO DE VASCONCELOS}

\section{REFERÊNCIAS}

ADNER, R.; HELFAT, C. E. Corporate effects and dynamic managerial capabilities. Strategic Management Journal, v. 24, n. 10, p. 1011-1025, 2003.

BAIN, J. Barriers to N ew Competition. Cambridge, MA: Harvard University Press, 1956.

BONARDI, J.; HILLMAN, A. J.; KEIM, G. D. The attractiveness of political markets: implications for firm strategy. Academy of M anagement Review, $v$. 30, n. 2, p. 397-413, abr. 2005.

BOW MAN, E. H; HELFAT, C. E. Does corporate strategy matter? Strategic Management Journal, v. 22, n. 1, p. 1-23, jan. 2001.

BRITO, L. A. L. Size and financial performance: a multi-level approach. ACADEMY OF MANAGEMENT MEETING, 2006, Atlanta, Proceedings. Academy of Management, 2006. 1 CD-ROM.

BRITO, L. A. L.; VASCONCELOS, F. C. How much does country matter? In: ENCONTRO DA ASSOCIAÇÃO NACIONAL DOS PROGRAMAS DE PÓS-GRADUAÇÃO EM ADMINISTRAÇÃO, 27., 2003, Atibaia. Anais. Atibaia: ANPAD, 2003. 1 CD-ROM.

BRITO, L. A. L.; VASCO N CELOS, F. C. Firm performance in Latin America: a different game? In: ENCONTRO DA ASSOCIAÇÃO NACIONAL DOS PROGRAMASDE PÓS-GRADUAÇÃO EM ADMINISTRAÇÃO, 27., 2004, Curitiba. Anais. Rio de Janeiro: ANPAD, 2004. 1 CD-ROM.

BROUTHERS, L. E. Explaining MNC profitability: country-specific, industry-specific and country-industry interactive influences. Management International Review, v. 38, n. 4, p. 345-361, 1998.

BROWN, P. R.; SOYBEL, V. E.; STICKNEY, C. P. Comparing U.S. and Japanese corporate-level operating performance using financial statement data. Strategic M anagement Journal, v. 15, n. 1, p. 75-83, 1994.

BRUSH, T. H.; BROMILEY, P. What does a small corporate effect mean? A variance-components simulation of corporate and business effects. Strategic Management Journal, v. 18, n. 10, p. 825-835, 1997.

BRUSH, T. H.; BROMILEY, P.; HENDRICKX, M. The relative influence of industry and corporation on business segment performance: an alternative estimate. Strategic M anagement Journal, v. 20, n. 6, p. 519-547, 1999.

CHRISTMAN, P.; DAY, D. L.; YIP, G. S. The relative influence of country conditions, industry structure and business strategy on MNC subsidiary performance. Journal of International Management, v. 5, n. 4, p. 241-265, 1999.

COLLINS, J. M. A market performance comparison of U.S. firms active in domestic, developed, and developing countries. Journal of International Business Studies, v. 21, n. 2, p. 271-287, 1990.
DIERICKX, I.; COOL, K. Asset stock accumulation and sustainability of competitive advantage. Management Science, v. 35, n. 12, p. 15041511, 1989.

EGGERTSSON, T. Economic Behavior and Institutions. Cambridge, MA: Cambridge University Press, 1990.

FOSS, K.; FOSS, N. J. Assets, attributes and ownership. International Journal of the Economics of Business, v. 8, p. 19-37, 2001.

FOSS, K.; FOSS, N. J. Resources and transaction costs: how property rights economics furthers the resource-based view. Strategic M anagement Journal, v. 26, n. 6, p. 541-553, 2005.

FURMAN, J. Does industry matter differently in different places? Evidence from four OECD countries. MIT - Sloan Working Paper, n. 4121, 2000.

GARELLI, S. Competitiveness of nations: the fundamentals. IMD World Competitiveness Yearbook, 2006

GHEMAWAT, P. Semiglobalization and international business strategy. Journal of International Business Studies, v. 34, n. 2, p. 138-152, mar. 2003.

HAAKE, S. National business systems and industry-specific competitiveness. Organization Studies, v. 23, n. 5, p. 711-736, 2002.

HALL, P. A.; SOSKICE, D. An introduction to varieties of capitalism. In: HALL, P. A.; SOSKICE, D. Varieties of Capitalism. The Institutional Foundations of Comparative Advantage. Oxford: Oxford University Press, 2001.

HAWAWINI, G.; SUBRAMANIAN, V.; VERDIN, P. Is performance driven by industry - or firm - specific factors? Strategic Management Journal, v. 24, n. 1, p. 1-16, jan. 2003.

HAWAWINI, G.; SUBRAMANIAN, V.; VERDIN, Paul. The home country in the age of globalization: how much does it matter for firm performance? Journal of World Business, v. 39, n. 2, p. 121-135, 2004.

HOUGH, J. R. Business segment performance redux: a multilevel approach. Strategic M anagement Journal, v. 27, n. 1, p. 45-61, 2006.

KIM, J.; MAHONEY, J. T. Property rights theory, transaction costs theory and agency theory: an organizational economics approach to strategic management. Managerial and Decision Economics, v. 26, n. 4, p. 223242, 2005

KO GUT, B. Country capabilities and the permeability of borders. Strategic Management Journal, v. 12, p. 33-47, 1991.

MAHMOOD, I. P.; RUFIN, C. Government's dilemma: the role of government in imitation and innovation. Academy of Management Review, v. 30, n. 2, p. 338-360, 2005. 
MAKINO, S.; BEAMISH, P. W.; ZHAO, N. B. The characteristics and performance of Japanese FDI in less developed and developed countries. Journal of World Business, v. 39, n. 4, p. 377-392, 2004.

MAKINO, S.; ISOBE, T.; CHAN, C. M. Does country matter? Strategic Management Journal, v. 25, n. 10, p. 1027-1043, 2004.

MCGAHAN, A. M.; PORTER, M. E. How much does industry matter, really? Strategic M anagement Journal, v. 18, p. 15-30, 1997.

MCNAMARA, G.; VAALER, P. M.; DEVERS, C. Same as it ever was: the search for evidence on increasing hypercompetition. Strategic $M$ anagement Journal, v. 24, n. 3, p. 261-278, 2003.

MEYERS, J. L. The impact of the inappropriate modelling of cross-classified data structures. Tese (Doutorado). Austin: University of Texas at Austin, Texas, USA, 2004.

MISANGYI, V. F.; ELMS, H.; GRECKHAMER, T.; LEPINE, J. A new perspective on a fundamental debate: a multilevel approach to industry, corporate, and business unit effects. Strategic Management Journal, v. 27, n. 6, p. $571-590,2006$ a.

MISANGYI, V.; LEPINE, J. A.; ALGINA, J.; GOEDDEKE Jr. F. The adequacy of repeated-mesures regression for multilevel research. Organizational Research Methods, v. 9, n. 1, p. 5-28, 2006b.

NORTH, D. C. Institutions, Institutional Change and Economic Performance. New York: Cambridge University Press, 1990.

PEDHAZUR, E. J.; SCHMELKIN, L. P. M easurement, D esign and Analysis. Hillsdale, NJ: Lawrence Erlbaum Associates, 1991.

PENG, M. W. Towards an institution-based view of business strategy. Asia Pacific Journal of Management, v. 19, n. 2, p. 251-268, 2002.

PETERAF, M. A. The cornerstones of competitive advantage: a resourcebased view. Strategic Management Journal, v. 14, n. 3, p. 179-191, 1993.

PORTER, M. E. Competitive Strategy. N ew York: The Free Press, 1980.

PORTER, M. E. The Competitive Advantage of Nations. New York: The Free Press, 1990.

PORTER, M. E. Building the microeconomic foundations of prosperity: findings from the business competitiveness index. In: WORLD COMPETITIVENESS REPORT 2003/2004. Disponível em: «ttp:॥ www. isc.hbs.edu/pdf/GCR_0203_mci.pdf >. Acesso em: 02 jan. 2007.

RAM OS-RODRÍGUEZ, A. R.; RUIZ-NAVARRO, J. Changes in the intellectual structure of strategic management research: a bibliometric study of the
Strategic M anagement Journal, 1980-2000. Strategic M anagement Journal, v. 25, n. 10, p. $981-1004,2004$.

RAUDENBUSH, S. W.; BRYK, A. S. Hierarchical Linear Models: Applications and Data Analysis Methods. 2. Ed. Thousand Oaks, CA: Sage, 2002.

RAUDENBUSH, S. W.; WILLMS, J. D. The estimation of school effects. Journal of Educational and Behavioral Statistics, v. 20, n. 4, p. 307-335, 1995

RING, P. S.; BIGLEY, G. A.; D'AUN N O, T.; KHANNA, T. Perspectives on how governments matter. Academy of Management Review, v. 30, n. 2, p. 308-320, 2005.

RODRIGUEZ, P.; UHLENBRUCK, K.; EDEN, L. Government corruption and the entry strategies of multinationals. A cademy of $M$ anagement Review, v. 30, n. 2, p. 383-396, 2005.

ROQUEBERT, J. A.; PHILLIPS, R. L.; WESTFALL, P. A. Market vs. management: what 'drives' profitability? Strategic Management Journal, v. 17, n. 8, p. 653-664, 1996.

RUEFLI, T. W.; WIGGINS, R. R. Industry, corporate and segment effects and business performance: a non-parametric approach. Strategic $M$ anagement Journal, v. 24, n. 9, p. 861-879, 2003.

RUMELT, R. P. How much does industry matter? Strategic Management Journal, v. 12, n. 3, p. 167-185, 1991.

SCHMALENSEE, R. Do markets differ much? American Economic Review, v. 75, n. 3, p. 341-351, 1985.

SHORT, J. C.; KETCHEN JR., D. J.; BEN NETT, N.; DU TOIT, M. An examination of firm, industry, and time effects on performance using random coefficients modeling. Organizational Research Methods, v. 9, n. 3, p. 259-284, 2006.

SHORT, J. C., KETCHEN JR., D. J., PALMER, T. B.; HULT, G. T. M. Firm, strategic group, and industry influences on performance. Strategic Management Journal, v. 28 n. 2, p. 147-167, 2007.

SIN GER, J. D.; WILLET, J. B. Applied Longitudinal Data Analysis: Modelling Change and Event Ocurrence. Oxford: Oxford University Press, 2003.

SNIJDERS, T. A. B.; BOSKER, R. J. Multilevel Analysis: An introduction to basic and advanced multilevel modeling. London: Sage Publications, 1999.

SPENCER, J. W.; MURTHA, T. P; LENWAY, S. A. How governments matter to new industry creation. Academy of M anagement Review, v. 30, n. 2, p. 321-337, 2005. 
STANDARD \& POOR'S. Research insight Compustat Global. Englewood, CO: McGraw-Hill, 2006 CD-ROM.

THOMAS, L; WARING, G. Competing capitalisms: capital investment in American, German, and Japanese firms. Strategic Management Journal, v. 20, n. 8, p. 729-748, 1999.

VICTER, R.; MCGAHAN, A. The effect of industry and location on firm profitability in the global market: empirical evidence that firm performance depends on the interaction between industry affiliation and country identity. DRUID SUM MER CONFEREN CE, 2006, Proceedings. DRUID, 2006.
WAHEEDUZZAMAN, A. N. M; RYANS JR., J. K. Definition, perspectives and understanding of international competitiveness: a quest for a common ground. Competitiveness Review, v. 6, n. 2 , p. 7-26, 1996.

WAN, W. P. Country resource environments, firm capabilities, and corporate diversification strategies. Journal of Management Studies, v. 42, n. 1, p.161-182, 2005.

WERNERFELT, B. A resource-based view of the firm. Strategic M anagement Journal, v. 5, n. 2, p. 171-180, 1984.

\section{Artigo recebido em 04.05.2007. A provado em 20.08.2007.}

\section{Rafael G uilherme Burstein Goldszmidt}

Doutorando em Administração de Empresas pela FGV-EAESP.

Interesses de pesquisa nas áreas de estratégia e métodos quantitativos.

E-mail: rafael.goldszmidt@fgv.br

Endereço: Rua Itapeva, 474, 90 andar, Bela Vista - São Paulo - SP, 01332-000.

\section{Flávio C arvalho de Vasconcelos}

Doutor em Administração de Empresas pela École des Hautes Études Commerciales (HEC).

Interesses de pesquisa nas áreas de estratégia e organizações.

E-mail: flavio.vasconcelos@fgv.br

Endereço: Rua Itapeva, 474, 11ํandar, Bela Vista, São Paulo - SP, 01332-000.

\section{Luiz Artur Ledur Brito}

Doutor em Administração de Empresas pela FGV-EAESP.

Interesses de pesquisa nas áreas de estratégia e operações.

E-mail: luiz.brito@fgv.br

Endereço: Rua Itapeva, 474, 8o andar, Bela Vista, São Paulo - SP, 01332-000. 